\title{
Predictive value of exhaled nitric oxide in the management of asthma: a systematic review
}

\author{
Lauri Lehtimäki ${ }^{1,2}$, Péter Csonka ${ }^{3}$, Eeva Mäkinen ${ }^{4}$, Jaana Isojärvi ${ }^{4}$, \\ Sirpa-Liisa Hovi ${ }^{4}$ and Anneli Ahovuo-Saloranta ${ }^{4}$
}

\author{
Affiliations: \\ ${ }^{1}$ Medical School, University of Tampere, Tampere, Finland. \\ ${ }^{2}$ Allergy Centre, Tampere University Hospital, Tampere, Finland. \\ ${ }^{3}$ Terveystalo Healthcare Oy, Tampere, Finland. \\ ${ }^{4}$ National Institute for Health and Welfare, Helsinki, Finland.
}

\section{Correspondence:}

Lauri Lehtimäki, University of Tampere, Medical School, FIN 33014 University of Tampere, Tampere, Finland. E-mail: lauri.lehtimakiduta.fi

ABSTRACT The clinical value of measuring fractional exhaled nitric oxide ( $F$ eNO) in asthma is not clear. We aimed to assess whether FeNO can reliably predict clinical outcomes in asthma treated with inhaled corticosteroids (ICS). We also evaluated whether its predictive role is influenced by different inflammatory phenotypes of asthma.

We conducted a systematic review focusing on five clinically relevant questions. Two authors independently screened search results, extracted data and assessed quality of the included studies. Data were synthesised by qualitative methods.

12 prospective studies were included, answering partly three of the five questions. In steroid-naïve asthma, a high FeNO level probably predicts good response to ICS. In ICS-treated asthmatics, a low FeNO level probably predicts low risk of exacerbation, and the patient is unlikely to benefit from increasing ICS dose. There were scarce data to conclude whether FeNO predicts exacerbations when ICS treatment is stopped in well-controlled asthma. Only one study reported results separately in different asthma phenotypes.

The current evidence on the predictive value of FeNO and its role in the management of asthma is incomplete. Future studies should focus on clinically meaningful questions and probably target only eosinophilic phenotypes where FeNO is best associated with the activity of airway inflammation.

@ERSpublications

Exhaled nitric oxide may predict clinical outcomes in asthma, but its role in different phenotypes is unclear http://ow.ly/bUDe301n4FN

This article has supplementary material available from erj.ersjournals.com

Received: April 062016 | Accepted after revision: June 032016 | First published online: Aug 042016

Conflict of interest: Disclosures can be found alongside this article at erj.ersjournals.com

Copyright OERS 2016 


\section{Introduction}

Asthma is characterised by chronic inflammation of the airways, bronchial hyperresponsiveness and recurrent symptoms. The degree and type of airway inflammation vary between individuals and multiple asthma phenotypes have been identified [1]. Eosinophilic inflammation is particularly pronounced in atopic asthma, but can be present in nonatopic phenotypes as well. Adult-onset asthma is sometimes characterised by neutrophilic inflammation or by almost nonexistent granulocyte activity [2]. Although inflammation is important in asthma, the diagnosis and follow-up of asthma are mainly based on symptoms and measures of lung function.

\section{Measurement of exhaled nitric oxide}

Airway inflammation is related to asthma symptoms and exacerbations [3,4], but it is difficult to measure directly the level and nature of inflammation in clinical practice. Fractional exhaled nitric oxide (FeNO) is particularly associated with eosinophilic airway inflammation [5-9]. Nitric oxide (NO) is an important cellular signalling molecule that regulates pulmonary blood flow, mucus production, ciliary activity and inflammation. Under normal circumstances $\mathrm{NO}$ is produced at very low concentrations by constitutive NO synthases (endothelial NOS and neuronal NOS). In cases of airway inflammation pro-inflammatory cytokines upregulate inducible NOS, producing higher levels of NO [10].

Using online methods, it is practically possible to measure FeNO in children aged $\geqslant 5$ years. In children, NO values are directly correlated with height, hence height-adjusted reference values are needed [11]. It is not known whether height significantly affects $\mathrm{NO}$ values in adults [12]. Active smoking reduces FeNO and corrupts the results. Atopic individuals have significantly elevated FeNO irrespective of asthma, both in children and adults [10], probably reflecting lower airway inflammation in the absence of airway hyperresponsiveness and clinical asthma. Other factors that influence FeNO values are respiratory infections, high dietary nitrate concentration, bronchial obstruction and, possibly, physical exercise [13].

There are international guidelines for FeNO measurement and interpretation $[12,13]$. As FeNO values vary with exhalation flow rate, it is generally recommended to measure FeNO during an exhalation of $\geqslant 6 \mathrm{~s}$ using $50 \mathrm{~mL} \cdot \mathrm{s}^{-1}$ flow rates and $5-20 \mathrm{cmH}_{2} \mathrm{O}$ of back pressure. FeNO levels $<25 \mathrm{ppb}$ in adults and $<20 \mathrm{ppb}$ in children are considered to be low, whereas with $F$ eNO $>50 \mathrm{ppb}$ in adults and $>35 \mathrm{ppb}$ in children the likelihood of eosinophilic inflammation is increased [12]. The same equipment and measurement procedures should be used for subsequent recordings and follow-up [13].

\section{What is the added value of FeNO measurement in asthma treatment?}

Asthma is a complex disease of the airways causing variable airflow obstruction and is associated with different types and intensities of airway inflammation [1]. Since there are no unequivocal and easily accessible methods for quantifying the inflammation per se, asthma diagnosis is mainly based on lung function measurements and identification of variable or reversible bronchoconstriction. FeNO measurement cannot supplant lung function tests, but as a marker of inflammation it might provide additional information for asthma diagnosis and estimating disease phenotype and activity. Since the basis of asthma therapy is to alleviate airway inflammation, FeNO could also predict or assess responsiveness to anti-inflammatory therapy.

\section{Study questions}

Previous reviews focusing on using $F$ eNO in the diagnosis of asthma or in adjusting individual corticosteroid treatment have been published $[14,15]$. The aim of this review was to assess the predictive value of FeNO measurements in asthma treated with inhaled corticosteroids (ICS). Concurrent use of other medication was allowed, but its effect was not evaluated.

We addressed five clinically relevant questions concerning the predictive utilisation of FeNO measurement:

1) Since glucocorticoids reduce eosinophilic inflammation more effectively than other types of inflammation [2], could high FeNO predict a favourable response to ICS treatment in steroid-naïve asthmatics?

2) During maintenance treatment, ICS dose should be kept as low as possible [1]. Could elevated FeNO identify those patients who are at risk of exacerbation?

3) During regular stable ICS treatment, could elevated FeNO identify those patients who would benefit from augmented glucocorticoid treatment?

4) If the patient is asymptomatic for a prolonged period of time with normal lung function, it is recommended to step-down controller treatment [1]? Does a low level of FeNO predict that ICS therapy could be successfully discontinued?

5) After cessation of ICS therapy, does an elevated FeNO predict the reactivation of the disease and hence the need for anti-inflammatory therapy? 
As the predictive ability of FeNO may depend on the inflammatory phenotype of asthma, in each of the study questions we addressed whether the inflammatory phenotype was defined and if the prognostic value of FeNO was assessed separately in different inflammatory phenotypes.

Methods

Criteria for including studies

Types of studies and participants

We included only prospective studies where the subjects were aged $\geqslant 5$ years (for reliable online FeNO measurement) and asthma was diagnosed based on reversible or variable airway obstruction [1]. The criteria for anti-inflammatory medication depended on the study question. Question 1: subjects had to be steroid-naïve; question 2: subjects had to be on regular stable ICS and additional oral glucocorticoids were allowed; question 3: subjects had to be on regular stable glucocorticoid treatment at baseline; and questions 4 and 5: at baseline subjects had to have good asthma control on low-dose ICS for $\geqslant 3$ months.

\section{Comparison of intervention and prognostic factors}

We included studies where high FeNO was compared to low FeNO; FeNO had to be measured using an online technique and measured at a healthcare unit excluding serial home measurements. Cut-off values for high and low FeNO were taken from each original study instead of setting predetermined values.

\section{Types of outcome measures}

The prognostic accuracy of FeNO to the following clinical outcomes had to be reported. 1) asthmatic symptoms, lung function or exacerbations of asthma (questions 1, 3, 4 and 5); and 2) exacerbations of asthma (question 2).

\section{Search strategy}

We searched the following six electronic databases up to September 24, 2015 to track down systematic reviews and individual trials: Medline (OVID), US National Library of Medicine PubMed, the Cochrane Database of Systematic Reviews and the Centre for Reviews and Dissemination (Health Technology Assessment Database, the NHS Economic Evaluation Database and the Database of Abstracts of Reviews of Effects). Search strategies are presented in online supplementary appendix 1. In addition to searching the electronic databases, we screened the reference lists from the already identified studies and review articles for any additional relevant studies.

There were no language restrictions. If the information in the report was insufficient to make the final assessment of inclusion or exclusion, we contacted the authors of the studies to obtain additional information.

\section{Data extraction and quality assessment}

At least two authors independently screened search results, extracted data and assessed risk of bias of the included studies. The following information was extracted from the included studies: study design, follow-up time, numbers of patients at baseline and analysed, age, sex, allergy, if asthma inflammatory phenotype was assessed, severity of asthma, forced expiratory volume in $1 \mathrm{~s}$ (FEV1) at baseline, exhalation flow rate $\left(\mathrm{mL} \cdot \mathrm{s}^{-1}\right)$ used in FeNO measurement, FeNO level at baseline and funding.

Assessments of the risk of bias in the studies were made by using the quality in prognostic studies (QUIPS) assessment tool [16]. We assessed the following six methodological domains: representativeness of study population; adequateness of follow-up period and attrition; appropriateness of prognostic factor $(\mathrm{FeNO})$ measurement; appropriateness of outcome measurement; adequateness of statistical analysis (including confounding factors) and reporting; and conflict of interests (detailed criteria for judgements are presented in online supplementary appendix 2). If the information in the report was insufficient to extract detailed data, we contacted the authors of studies to obtain additional information.

To draw conclusions about the overall risk of bias within a study, we decided to classify the studies into three categories: studies with low, unclear or high risk of bias. If all the domains within a study were graded as having low risk of bias, the overall judgement was low risk of bias, and if even one of the domains was assessed as having high risk of bias, the overall risk of bias for a study was graded as high risk of bias.

\section{Data synthesis}

The combined qualitative result for each research question is based on the similarity of the results of the individual studies and their overall assessments of risk of bias. Quantitative analyses were not made due to heterogeneity across the included studies at each research question regarding e.g. outcome measures. 


\section{Results}

Search results

In total 1846 records were identified from the electronic searches after removing duplicates. We discarded 1744 records as irrelevant to this review on the basis of abstract or title. We obtained the remaining 102 reports in full-text and among those we found 12 eligible prognostic accuracy studies (13 reports). No additional studies were identified by screening reference lists from the already identified studies and review articles. The processing of search results is presented as a flow chart in figure 1 according to the Preferred Reporting Items for Systematic Reviews and Meta-analyses statement [17]. The exclusion of the 89 full-text reports was decided in two stages: at the first stage, 81 reports were excluded mainly because studies considered only asthma diagnostics, or the design or research question of studies clearly did not fulfill the inclusion criteria of this review; at the final stage, eight further studies were excluded. Detailed reasons for their exclusion are presented in online supplementary appendix 3.

\section{Characteristics of included studies}

Detailed characteristics of the 12 included studies are provided in online supplementary appendix 4 . All study designs were prospective cohort studies related to FeNO measurement. The number of participants included in the analyses of prognostic accuracy varied from 21 to 169 in adults (nine studies in adults), and from 28 to 111 in children (three studies in children). The mean or median age ranged from 30 years to 56 years in adults, and was on average 11 years in children. Studies in adults included participants with asthma of varying severity from mild to severe, but not all the studies reported the severity of asthma. Most of the studies measured FeNO using the recommended flow rate of $50 \mathrm{~mL} \cdot \mathrm{s}^{-1}$, but one study used a higher flow rate of $100 \mathrm{~mL} \cdot \mathrm{s}^{-1}$ and two did not report the flow rate used. There was marked variation in baseline FeNO between the studies, reflecting differences in factors affecting FeNO such as age, use of anti-inflammatory medication and possibly differences in phenotypes of asthma. Seven out of the 12
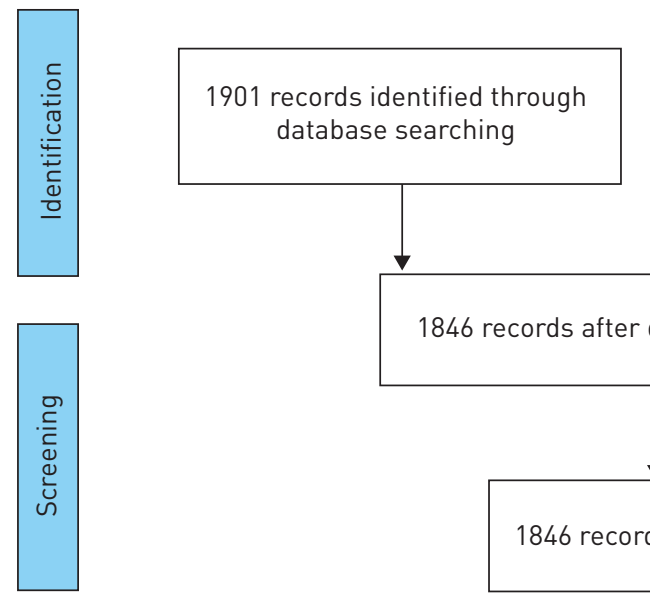

$$
\text { through other sources }
$$

0 additional records identified
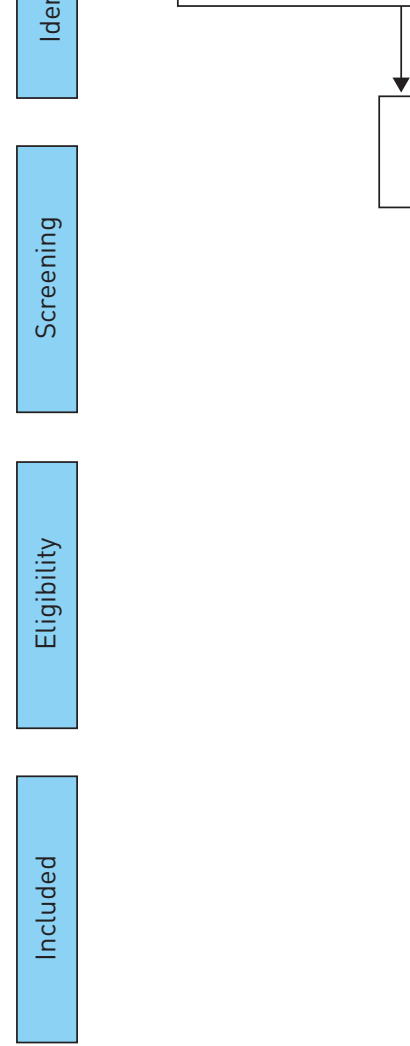

846 records after duplicates removed

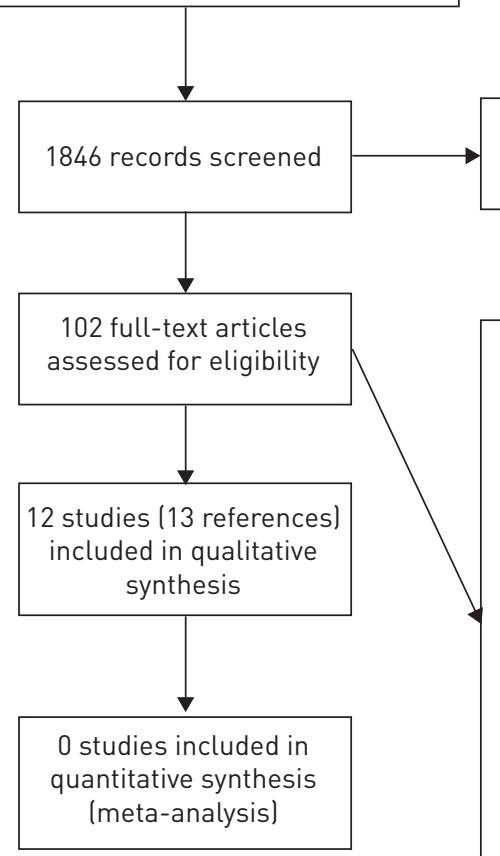

1744 records discarded

\section{At first stage:}

81 full-text articles excluded Istudies considered only asthma diagnostics or the design or research question of studies clearly did not fulfill the inclusion criteria of this review)

At the final stage: 8 studies excluded Iminor deviation from the inclusion criteria of this review] 
studies were supported by national or academic sources without or with equipment support by a manufacturer, and one study was supported by a manufacturer which did not participate in the data collection, analysis or writing of the manuscript. The other four studies did not provide information on funding or support.

$10(83 \%)$ out of the 12 studies showed unclear or high risk of bias in at least one domain, often because of a high drop-out rate or incomplete blinding of the outcome measurement or a follow-up time too short for an outcome. Thus, only two studies were assessed as having low overall risk of bias [18, 19]. Detailed results of the risk of bias assessments are provided in online supplementary appendix 5.

\section{Predictive accuracy}

Does increased FeNO predict a favourable response to ICS in steroid-naïve asthmatics?

In steroid-naïve patients with asthma, increased FeNO probably predicts favourable response to ICS (two studies in adults and one in children; one with low risk and two with high risk of bias; the results are similar between the studies).

In a 24-week study $(\mathrm{n}=21)$ by SzEFLER et al. [20] in 2002, the baseline FeNO was significantly higher in those adult asthmatics in whom treatment with ICS increased FEV $1 \geqslant 15 \%$ compared to those in whom FEV 1 increased $<5 \%$ (median (interquartile range (IQR)) $17.6 \mathrm{ppb}$ (16.1-23.0 ppb) versus $11.1 \mathrm{ppb}$ (7.9$14.2 \mathrm{ppb}$ ); exhalation flow rate not reported). In another study in adult asthmatics ( $\mathrm{n}=88)$, CowAN et al. [18] reported that high baseline FeNO $\left(>33 \mathrm{ppb}\right.$, flow rate $\left.50 \mathrm{~mL} \cdot \mathrm{s}^{-1}\right)$ predicted better symptom alleviation during ICS treatment of $\geqslant 4$ weeks in eosinophilic asthma and decrease of bronchial hyperresponsiveness in both eosinophilic and noneosinophilic asthma. A further subanalysis of the same study focusing on different inflammatory markers confirmed the results on the predictive ability of FeNO [21].

In another study by SzEFLER et al. [22] in 2005, $40 \%$ of asthmatic children ( $\mathrm{n}=111$, mean age 11 years), responded to ICS treatment lasting 8 weeks, response defined as improvement in $\mathrm{FEV} 1$ of $\geqslant 7.5 \%$. Univariable analysis with dichotomised predictors revealed that one of the significant predictors of a response to ICS treatment included baseline FeNO $>25 \mathrm{ppb}$ (flow rate $50 \mathrm{~mL} \cdot \mathrm{s}^{-1}$ ) $(\mathrm{OR} 2.8, \mathrm{p}<0.05)$.

\section{Does increased FeNo predict risk of exacerbation in asthmatic patients on regular stable ICS} treatment?

Based on three studies in adults (one with high risk of bias and the other two with unclear risk) there is indication that low FeNO in an asthmatic subject on regular ICS treatment predicts a low risk of exacerbation, while high FeNO predicts a high risk of exacerbation.

Gelb et al. [23] studied whether high FeNO predicted exacerbations during the forthcoming 18 months in stable nonsmoking adult asthmatic patients $(n=44)$ on regular ICS plus long-acting $\beta$-agonist treatment. Half the patients ( 22 out of 44 ) had at least one exacerbation during follow-up, and $59 \%$ of these (13 out of 22) had baseline FeNO $\geqslant 28 \mathrm{ppb}$. In subjects with baseline $\mathrm{FeNO}>28 \mathrm{ppb}$ (flow rate $100 \mathrm{~mL} \cdot \mathrm{s}^{-1}$ ), $77 \%$ had an exacerbation (positive predictive value (PPV) 77\%), while in subjects with $\mathrm{FeNO}<28 \mathrm{ppb}, 87 \%$ did not have an exacerbation (negative predictive value (NPV) 87\%).

OzIER et al. [24] studied whether FeNO can be used to predict maintenance of good asthma control during a 3-week follow-up in 55 adults with well-controlled asthma (41 on ICS, 14 without ICS). A low baseline FeNO $<31 \mathrm{ppb}$ (flow rate $50 \mathrm{~mL} \cdot \mathrm{s}^{-1}$ ) was a good predictor of not losing good asthma control (NPV 95.3\%), but high FeNO $>31$ ppb was only a modest predictor of loss of asthma control (PPV 66.7\%).

KUPCZYK et al. [25] followed 169 adult asthmatics on regular ICS (76 mild-to-moderate asthmatics on a low-to-medium dose of ICS and 93 severe asthmatics on high doses of ICS with or without oral glucocorticoids) for 1 year. 104 exacerbations were recorded in severe asthmatics and 18 in mild-to-moderate asthmatics. Baseline $F$ eNO $>45 \mathrm{ppb}$ was associated with an increased risk of having at least two exacerbations in the whole group (OR (95\% CI) 4.32 (1.02-18.31), p=0.047; adjusted for age, sex, smoking, asthma severity and atopy) and in the severe asthma group (OR (95\% CI) 5.98 (1.28-27.99), $\mathrm{p}=0.023$; adjusted for age, sex, smoking and atopy).

\section{Does increased FeNO in asthmatic patients on regular stable ICS treatment predict further benefit} from augmenting the glucocorticoid treatment?

We found four studies addressing this question. Two of these studies [19, 26] reported that increased FeNO in asthmatic subjects on regular ICS predicts further benefit from enhancing the glucocorticoid treatment, but this was not supported by the other two studies [27, 28]. However, all four studies reported that low FeNO in asthmatics on regular ICS implies that enhancing the glucocorticoid dose would not result in improvement of asthma (one study with high risk of bias, one with low risk and two with unclear risk of bias). 
PÉREZ-DE-LlANo et al. [26] found that among 102 asthmatics with poor control of their disease, baseline FeNO $>30 \mathrm{ppb}$ (flow rate $50 \mathrm{~mL} \cdot \mathrm{s}^{-1}$ ) predicted that the patient gained from enhancing glucocorticoid treatment by increasing the dose of ICS and, if further needed, by adding oral glucocorticoids (PPV $87.5 \%$ ), while $F \mathrm{eNO}<30 \mathrm{ppb}$ predicted no improvement (NPV 90.6\%).

LiTTLE et al. [19] started oral prednisolone (20 mg per day) on 37 asthmatics (36 already on ICS) for 2 weeks. Baseline FeNO $>10 \mathrm{ppb}$ (flow rate not reported) predicted that the subject would gain an increase $>15 \%$ in FEV1 (PPV 83\%), while FeNO < 10 ppb probably excluded such an increase (NPV 72\%).

In 2013 KuPCZYK et al. [27] provided results for this study question based on the same study population as in their study described earlier [25], but drop-outs were excluded from the analyses in this report. This report included subjects with severe $(n=84)$ and mild-to-moderate $(n=62)$ asthma who were treated for 2 weeks with oral prednisolone in addition to their regular treatment. In subjects with severe asthma, baseline FeNO $<45 \mathrm{ppb}$ (flow rate $50 \mathrm{~mL} \cdot \mathrm{s}^{-1}$ ) excluded a favourable response (defined as improvement of $\geqslant 12 \%$ in $\mathrm{FEV}$; NPV 92.0\%), but high FeNO $>45 \mathrm{ppb}$ was not a reliable predictor of favourable response (PPV 31.2\%). In mild-to-moderate asthma, baseline FeNO was not a significant predictor of response in FEV1.

MichiLs et al. [28] studied 82 asthmatics with poor asthma control on regular ICS treatment of $\geqslant 500 \mu \mathrm{g}$ of beclometasone (25 also used leukotriene receptor antagonists (LTRAs) and six were on oral prednisolone). Baseline FeNO <35 ppb (flow rate $50 \mathrm{~mL} \cdot \mathrm{s}^{-1}$ ) probably excluded the symptomatic benefit (NPV $88 \%$ ) of increasing the ICS dose (13 subjects were started on LTRA simultaneously and one with oral prednisolone), but $\mathrm{FeNO}>35 \mathrm{ppb}$ was not a reliable predictor of favourable response (PPV 42\%).

If an asthmatic patient on low-dose ICS has good asthma control for $\geqslant 3$ months, does low baseline FeNo predict successful withdrawal of ICS without asthma relapse?

There are insufficient data to conclude whether or not low FeNO predicts that the patient could be weaned off ICS without risk of activation of asthma (one small study in children with unclear risk of bias).

Pijnenburg et al. [29] followed 37 asthmatic children (mean (range) age 12 (7-17) years) without asthmatic symptoms during the prior 6 months while on regular ICS treatment (100-400 $\mu$ g budesonide or equivalent per day). FeNO was measured at baseline and at control visits 2, 4, 12 and 24 weeks after discontinuing the treatment. Nine children had a relapse (median (range) 36 (14-141) days after discontinuation). There was no significant difference in baseline FeNO between those who relapsed and those who did not (geometric mean 14.8 ppb versus $10.5 \mathrm{ppb}$; ratio (95\% CI) 1.4 (0.7-2.8), p=0.32). The mean age and height of the children with and without relapse were similar.

If an asthmatic patient on low-dose ICS has good asthma control for $\geqslant 3$ months and the ICS is withdrawn, does increased FeNo level during the follow-up predict asthma relapse?

There are insufficient data to conclude whether or not increased FeNO level after discontinuing ICS treatment predicts asthma relapse (two small studies in children with conflicting results; both with unclear risk of bias).

In the study by Pijnenburg et al. [29] described earlier, FenO levels measured 2 and 4 weeks after discontinuing ICS were higher in those children who relapsed than in those who did not (geometric mean FeNO at 2 weeks $35.3 \mathrm{ppb}(\mathrm{n}=8)$ versus $15.7 \mathrm{ppb}$; ratio (95\% CI) $2.3(1.2-4.1), \mathrm{p}=0.01$; at 4 weeks $40.8 \mathrm{ppb}$ $(\mathrm{n}=7)$ versus $15.9 \mathrm{ppb}$, ratio $(95 \% \mathrm{CI}) 2.6(1.3-5.1), \mathrm{p}=0.009)$. However, even among children with increasing FeNO, the majority of children did not relapse in 24 weeks of follow-up.

FERRER et al. [30] recruited asthmatic children (mean (range) age 11 (6-14) years) in whom regular asthma treatment had been stopped 1-12 months previously and who had good asthma control (their previous asthma medication is not reported). FeNO was measured and the children were divided into two groups having FeNO either above or below $49 \mathrm{ppb}$. After 6 months follow-up, five out of 15 children with baseline FeNO $<49$ ppb had relapsed, while only one out of 13 children with baseline FeNO $>49$ ppb relapsed. The difference in risk of relapse between the groups was not statistically significant (risk ratio (95\% CI) 0.2 $(0.03-1.72))$. The cut-off value was based on the median FeNO value in this study and on the results reported by Pijnenburg et al. [29]. Due to the relatively small sample size, this cut-off value cannot be considered very reliable. However, the predictive value of FeNO in the present study would have been roughly the same even with different cut-off levels, as the FeNO values in children with a relapse were widely scattered $(13,14,25,33,34$ and $60 \mathrm{ppb})$.

The predictive ability of FeNO in different inflammatory phenotypes

Out of the 12 studies included, six reported some measures of inflammatory activity and type (e.g. sputum or blood eosinophils) and three of these studies categorised subjects into eosinophilic or noneosinophilic phenotypes (online supplementary appendix 4). However, only one study (Cowan et al. [18]) assessed the 
predictive value of FeNO separately in different inflammatory phenotypes (discussed earlier). The rest of the studies did not take inflammatory phenotype into account in the analysis.

\section{Discussion}

There is increasing interest in noninvasive measures of inflammation to guide asthma management. Prognostic studies analysed in this review measure the accuracy of FeNO to predict clinical outcomes and not the benefit in patient management directly. Based on these data, it seems that in steroid-naïve patients, high FeNO levels predict good response to ICS treatment. A low level of FeNO in adult patients on regular ICS therapy is associated with reduced likelihood of exacerbation, and these patients are less likely to benefit from increased doses of ICS, regardless of possible symptoms. Currently, there are not enough data to conclude whether or not FeNO can predict the risk of exacerbation after ICS reduction or cessation in well-controlled asthma.

The number of included studies in each research question in this review was small and, hence, it is difficult to draw firm conclusions. Furthermore, although we included only prospective designs, the information was mainly of poor quality with studies having small samples and unclear or high overall risk of bias. In addition, the studies comprised different designs, medication protocols, FeNO cut-off values, visit intervals, lengths of follow-up, definitions of asthma exacerbation and inclusion criteria of patients. Furthermore, it has been shown that in children FeNO values are related to age and height and increase linearly between 6 and 14 years of age in females and between 6 and 16 years of age in males [11, 31]. This is an important factor that may have influenced the ability of FeNO to predict outcomes in children, as the studies in this review included children of quite wide age ranges.

When first reports of increased FeNO in subjects with asthma were published more than 20 years ago [32, 33], our understanding of asthma was not as broad as it is nowadays. Asthma was mainly divided into intrinsic and extrinsic types and both were largely considered as eosinophilic [34]. It is well established that FeNO increases most markedly in T-helper type 2 lymphocyte-driven eosinophilic airway inflammation [35], and that this elevation can be readily reduced by ICS therapy. Therefore, FeNO was studied as a potential "inflammometer" in asthma in general and not in certain subtypes of asthma. However, a wide spectrum of different phenotypes of asthma have been recognised [2], some of which are characterised by neutrophilic rather than eosinophilic inflammation, while others may not have mucosal inflammation at all. These noneosinophilic phenotypes of asthma are probably not associated with FeNO level. In the present systematic review, only half of the studies assessed inflammatory activity and phenotype in some way and a quarter of the studies assessed the proportion of subjects with eosinophilic asthma in their sample. Furthermore, only one study evaluated the ability of FeNO to predict responsiveness to ICS separately in eosinophilic and noneosinophilic asthma [18]. In that study, elevated baseline FeNO predicted improvement of asthma control only in eosinophilic asthma, but alleviation of bronchial hyperresponsiveness in both eosinophilic and noneosinophilic asthma. One reason for inconsistence between the results of different studies might be that the studies did not focus on certain NO-related asthma phenotypes, but recruited asthmatics in general. FeNO might be suitable for "FeNO-typing" asthma and it might be more useful for clinical decision making if applied only in eosinophilic phenotypes that are usually associated with higher FeNO.

We found that a low level of FeNO in adult patients on regular ICS therapy is associated with reduced likelihood of exacerbation, and these patients are less likely to benefit from increased doses of ICS. These results suggest that FeNO could be used in tailoring individual ICS dosage and help in directing ICS to those subjects with active steroid-sensitive eosinophilic inflammation. This clinical issue has been addressed by several studies and the information has been summarised (e.g. in a recently published Health Technology Assessment report [14] and in a meta-analysis [15]). According to these reports the evidence is inconclusive, although consistent with FeNO use resulting in fewer exacerbations, with a small or zero reduction in ICS use in adults and a possible increase in ICS use in children or patients with more severe asthma. In a way, our research questions on the predictive ability of $F$ eNO are a part of this broader question of using FeNO as a monitoring tool. However, none of the studies assessing the ability of FeNO to guide individual ICS dose adjustment focused on FeNO-related asthma types, but instead asthmatics in general were included. In addition to differences in treatment algorithms and cut-off values, this heterogeneity of asthma phenotypes is likely to be a confounding factor, possibly weakening the clinical benefit of FeNO measurements. In treatment-tailoring studies, FeNO should probably not be evaluated as a replacement for symptom assessment and lung function measurement, but rather as a complementary tool.

The utility of FeNO in the diagnosis of asthma was not evaluated in the current review. As long as the definition of asthma remains tightly linked to the detection of variable or reversible airway obstruction, markers of inflammation cannot replace lung function measurements, but they could be augmenting tools providing information on the other major aspect of asthma pathophysiology. Now that new markers of type and activity of airway inflammation are available and new biological treatments focus on certain 
mediators of inflammation, it may be that the whole concept of asthma needs to be revised. Currently, suitable patients for ICS treatment in the clinic are mainly identified based on symptoms and variable lung function, but revision of the diagnostic label "asthma" might help us to better aim ICS at subjects with steroid-sensitive inflammation, and also ultimately to identify subjects in whom the treatment should be based on other drug classes. High FeNO has been associated with better response to treatment with anti-IgE or anti-interleukin-13 antibodies [36, 37].

Measuring FeNO is technically simple, relatively economical and safe. However, there might be a risk of under- or overtreatment of asthma if clinical decisions rely too heavily on FeNO values.

Based on this review, it is not possible to provide clear recommendation about the use of FeNO in asthma management. It is difficult to provide exact cut-off values for clinicians due to heterogeneity of cut-off values and exhalation flow rates used across studies. It is possible that instead of predetermined absolute cut-off levels, individual values relative to personal best value might be more useful. How frequently $F$ eNO should be measured; how far in time we can extrapolate FeNO values measured at a certain time point; and which patient groups are more likely to benefit from FeNO measurements also remain uncertain.

In conclusion, there is a lack of good-quality data to draw firm conclusions on the predictive role of FeNO in asthma. It seems that in steroid-naïve patients high FeNO predicts a good response to ICS, while low FeNO in adult patients on regular ICS predicts reduced likelihood of exacerbation and no benefit from increased doses of ICS regardless of possible symptoms. Very little is known about how the predictive ability of FeNO depends on the inflammatory phenotype of asthma.

\section{Acknowledgements}

This review is based on work undertaken as part of a health technology assessment project (HALO project) guided by the National Institute for Health and Welfare in Finland. Part of the results has been published in Finnish (L. Lehtimäki et al. Uloshengitysilman typpioksidimittauksen merkitys astman glukokortikoidihoidossa. Finnish Med J 2015; 69: 45-49).

\section{References}

1 Global Initiative for Asthma (GINA). Global Strategy for Asthma Management and Prevention. GINA, 2015.

2 Wenzel SE. Asthma phenotypes: the evolution from clinical to molecular approaches. Nat Med 2012; 18: 716-725.

3 Ulrik CS. Peripheral eosinophil counts as a marker of disease activity in intrinsic and extrinsic asthma. Clin Exp Allergy 1995; 25: 820-827.

4 Belda J, Giner J, Casan P, et al. Mild exacerbations and eosinophilic inflammation in patients with stable, well-controlled asthma after 1 year of follow-up. Chest 2001; 119: 1011-1017.

5 Jatakanon A, Lim S, Kharitonov SA, et al. Correlation between exhaled nitric oxide, sputum eosinophils, and methacholine responsiveness in patients with mild asthma. Thorax 1998; 53: 91-95.

6 Berlyne GS, Parameswaran K, Kamada D, et al. A comparison of exhaled nitric oxide and induced sputum as markers of airway inflammation. J Allergy Clin Immunol 2000; 106: 638-644.

7 Payne DN, Adcock IM, Wilson NM, et al. Relationship between exhaled nitric oxide and mucosal eosinophilic inflammation in children with difficult asthma, after treatment with oral prednisolone. Am J Respir Crit Care Med 2001; 164: 1376-1381.

8 van den Toorn LM, Overbeek SE, de Jongste JC, et al. Airway inflammation is present during clinical remission of atopic asthma. Am J Respir Crit Care Med 2001; 164: 2107-2113.

9 Warke TJ, Fitch PS, Brown V, et al. Exhaled nitric oxide correlates with airway eosinophils in childhood asthma. Thorax 2002; 57: 383-387.

10 Sandrini A, Taylor DR, Thomas PS, et al. Fractional exhaled nitric oxide in asthma: an update. Respirology 2010; 15: $57-70$.

11 Malmberg LP, Petäys T, Haahtela T, et al. Exhaled nitric oxide in healthy nonatopic school-age children: determinants and height-adjusted reference values. Pediatr Pulmonol 2006; 41: 635-642.

12 Dweik RA, Boggs PB, Erzurum SC, et al. An official ATS clinical practice guideline: interpretation of exhaled nitric oxide levels (FENO) for clinical applications. Am J Respir Crit Care Med 2011; 184: 602-615.

13 American Thoracic Society, European Respiratory Society. ATS/ERS recommendations for standardized procedures for the online and offline measurement of exhaled lower respiratory nitric oxide and nasal nitric oxide, 2005. Am J Respir Crit Care Med 2005; 171: 912-930.

14 Harnan SE, Tappenden P, Essat $\mathrm{M}$, et al. Measurement of exhaled nitric oxide concentration in asthma: a systematic review and economic evaluation of NIOX MINO, NIOX VERO and NObreath. Health Technol Assess 2015; 19: 1-330.

15 Essat M, Harnan S, Gomersall T, et al. Fractional exhaled nitric oxide for the management of asthma in adults: a systematic review. Eur Respir J 2016; 47: 751-768.

16 Hayden JA, van der Windt DA, Cartwright JL, et al. Assessing bias in studies of prognostic factors. Ann Intern Med 2013; 158: 280-286.

17 Moher D, Liberati A, Tetzlaff J, et al. Preferred reporting items for systematic reviews and meta-analyses: the PRISMA statement. PLoS Med 2009; 6: e1000097.

18 Cowan DC, Cowan JO, Palmay R, et al. Effects of steroid therapy on inflammatory cell subtypes in asthma. Thorax 2010; 65: 384-390.

19 Little SA, Chalmers GW, MacLeod KJ, et al. Non-invasive markers of airway inflammation as predictors of oral steroid responsiveness in asthma. Thorax 2000; 55: 232-234.

20 Szefler SJ, Martin RJ, King TS, et al. Significant variability in response to inhaled corticosteroids for persistent asthma. J Allergy Clin Immunol 2002; 109: 410-418. 
21 Cowan DC, Taylor DR, Peterson LE, et al. Biomarker-based asthma phenotypes of corticosteroid response. J Allergy Clin Immunol 2015; 135: 877-883.

22 Szefler SJ, Phillips BR, Martinez FD, et al. Characterization of within-subject responses to fluticasone and montelukast in childhood asthma. J Allergy Clin Immunol 2005; 115: 233-242.

23 Gelb AF, Flynn Taylor C, Shinar CM, et al. Role of spirometry and exhaled nitric oxide to predict exacerbations in treated asthmatics. Chest 2006; 129: 1492-1499.

24 Ozier A, Girodet PO, Bara I, et al. Control maintenance can be predicted by exhaled NO monitoring in asthmatic patients. Respir Med 2011; 105: 989-996.

25 Kupczyk M, ten Brinke A, Sterk PJ, et al. Frequent exacerbators - a distinct phenotype of severe asthma. Clin Exp Allergy 2014; 44: 212-221.

26 Pérez-de-Llano LA, Carballada F, Castro Añón O, et al. Exhaled nitric oxide predicts control in patients with difficult-to-treat asthma. Eur Respir J 2010; 35: 1221-1227.

27 Kupczyk M, Haque S, Middelveld RJ, et al. Phenotypic predictors of response to oral glucocorticosteroids in severe asthma. Respir Med 2013; 107: 1521-1530.

28 Michils A, Baldassarre S, Van Muylem A. Exhaled nitric oxide and asthma control: a longitudinal study in unselected patients. Eur Respir J 2008; 31: 539-546.

29 Pijnenburg MW, Hofhuis W, Hop WC, et al. Exhaled nitric oxide predicts asthma relapse in children with clinical asthma remission. Thorax 2005; 60: 215-218.

30 Ferrer M, Jarque A, Tosca R, et al. Is it necessary to treat all asthmatic children with raised levels of exhaled nitric oxide?: treating the patient or the data. Allergol Immunopathol 2011; 39: 280-283.

31 Jacinto T, Malinovschi A, Janson C, et al. Evolution of exhaled nitric oxide levels throughout development and aging of healthy humans. J Breath Res 2015; 9: 036005.

32 Alving K, Weitzberg E, Lundberg JM. Increased amount of nitric oxide in exhaled air of asthmatics. Eur Respir J 1993; 6: 1368-1370.

33 Kharitonov SA, Yates D, Robbins RA, et al. Increased nitric oxide in exhaled air of asthmatic patients. Lancet 1994; 343: 133-135.

34 National Heart, Lung, and Blood Institute. Guidelines for the Diagnosis and Management of Asthma 1997. www.nhlbi.nih.gov/files/docs/guidelines/asthgdln_archive.pdf Date last updated: July 1997.

35 Bjermer L, Alving K, Diamant Z, et al. Current evidence and future research needs for FeNO measurement in respiratory diseases. Respir Med 2014; 108: 830-841.

36 Hanania NA, Wenzel S, Rosén K, et al. Exploring the effects of omalizumab in allergic asthma: an analysis of biomarkers in the EXTRA study. Am J Respir Crit Care Med 2013; 187: 804-811.

37 Corren J, Lemanske RF, Hanania NA, et al. Lebrikizumab treatment in adults with asthma. N Engl J Med 2011; 365: 1088-1098. 